\title{
Low invasive in vivo tissue sampling for monitoring biomarkers and drugs during surgery
}

\author{
Barbara Bojko ${ }^{1}$, Krzysztof Gorynski ${ }^{1}$, German A Gomez-Rios ${ }^{1}$, Jan M Knaak ${ }^{2}$, Tiago Machuca ${ }^{3}$, Erasmus Cudjoe ${ }^{1}$, \\ Vinzent N Spetzler ${ }^{2}$, Michael Hsin³ ${ }^{3}$ Marcelo Cypel ${ }^{3}$, Markus Selzner ${ }^{2}$, Mingyao Liư ${ }^{3}$, Shaf Keshjavee ${ }^{3}$ and \\ Janusz Pawliszyn ${ }^{1}$
}

The techniques currently used for drug, metabolite, and biomarker determination are based on sample collection, and therefore they are not suitable for repeated analysis because of the high invasiveness. Here, we present a novel method of biochemical analysis directly in organ during operation without need of a separate sample collection step: solid-phase microextraction (SPME). The approach is based on flexible microprobe coated with biocompatible extraction phase that is inserted to the tissue with no damage or disturbance of the organ. The method was evaluated during lung and liver transplantations using normothermic ex vivo liver perfusion (NEVLP) and ex vivo lung perfusion (EVLP). The study demonstrated feasibility of the method to extract wide range of endogenous compounds and drugs. Statistical analysis allowed observing metabolic changes of lung during cold ischemic time, perfusion, and reperfusion. It was also demonstrated that the level of drugs and their metabolites can be monitored over time. Based on the methylprednisolone as a selected example, the impairment of enzymatic properties of liver was detected in the injured organs but not in healthy control. This finding was supported by changes in pathways of endogenous metabolites. The SPME probe was also used for analysis of perfusion fluid using stopcock connection. The evaluation of biochemical profile of perfusates demonstrated potential of the approach for monitoring organ function during ex vivo perfusion. The simplicity of the device makes it convenient to use by medical personnel. With the microprobe, different areas of the organ or various organs can be sampled simultaneously. The technology allows assessment of organ function by biochemical profiling, determination of potential biomarkers, and drug monitoring. The use of this method for preintervention analysis could enhance the decision-making process for the best possible personalized approach, whereas post-transplantation monitoring would be used for graft assessments and fast response in case of organ failure.

Laboratory Investigation (2014) 94, 586-594; doi:10.1038/labinvest.2014.44; published online 31 March 2014

KEYWORDS: biomarker; drug metabolism; in vivo; liver; lung; metabolomics; solid phase microextraction

Monitoring of organ function during transplantation or intersurgery tissue analysis is a common approach in clinical practice. However, there are some limitations related to these procedures; for instance, chemical characterization is mainly performed from blood or urine samples because of the easy access to these matrices, whereas for histology studies, only a small piece of tissue is collected. Non- and low-invasive monitoring procedures are conducted by imaging techniques that do not give insight into the biochemistry of the organs. Nevertheless, sample collection is necessary in order to investigate changes in metabolic profiles, or estimate concentration of a drug or biomarker in the studied region (eg, cancer tissue). The most commonly used approach involves removing a piece of tissue either by using a biopsy needle or cutting a fragment of tissue during surgery. However, because of the risk of complications involved in invasive procedures, such as bleeding, these procedures cannot be repeated to monitor changes over time, or to collect samples from different organ regions (eg, different lobes of the liver or lung). Typically, biopsy tissue is subjected to histological examinations that evaluate the structure of the tissue and verify type, degree, and range of the abnormalities.

\footnotetext{
${ }^{1}$ Department of Chemistry, University of Waterloo, Waterloo, ON, Canada; ${ }^{2}$ Department of Surgery, Multi Organ Transplant Program, Toronto General Hospital, Toronto, ON, Canada and ${ }^{3}$ Latner Thoracic Surgery Research Laboratories, Toronto General Research Institute, University Health Network and Department of Surgery, University of Toronto, Toronto, ON, Canada

Correspondence: Professor J Pawliszyn, PhD, Department of Chemistry, University of Waterloo, 200 University Avenue West, Waterloo, ON N2L 3G1, Canada.

E-mail: janusz@uwaterloo.ca

Received 10 December 2013; accepted 2 February 2014
} 
More recently, techniques involving the tracking of biochemical alterations on a global view have been gaining popularity. These techniques can be used to find specific biomarkers or get information about the pathways corresponding to a given disease. Nicholson et al, ${ }^{1}$ in their review on metabolic phenotyping in clinical and surgical environments, emphasized several aspects of bringing this tool to clinics. The authors drew a picture of time-extended metabotyping of individual patients that would allow for diagnosis and prevention, assessment of disease progress, and effectiveness of the applied treatment. They also highlighted the lack of 'molecular diagnostic and prognostic instruments' in the surgical environment that is 'the most personalized therapeutic option' of treatment. The importance of metabolomics and monitoring of biomarkers in transplantation has been previously reviewed by Wishart ${ }^{2}$ in 2005; as is indicated by Wishart, ${ }^{2}$ the main advantage of metabolomics over genomics, transcriptomics, or proteomics is a timescale of occurring changes; the effect of stimuli on small molecules can be detected within seconds or minutes, whereas changes in protein level or profile or gene expression can only be observed within a matter of days or weeks.

The most common analytical platforms for this type of global profiling are mass spectrometers coupled to liquid or gas chromatographs and nuclear magnetic resonance spectrometers. However, these powerful instruments, and particularly mass spectrometers, require appropriate sample preparation before analysis. Several protocols describing tissue sample preparation for metabolomics, metabolic profiling, or fingerprinting have been proposed, but most of them are based on the removal of a piece of tissue, followed by labor-intensive and time-consuming homogenization, drying, and multisolvent extraction, ${ }^{3-5}$ therefore limiting their onsite use. To overcome this challenge and provide a tool where direct untargeted analysis of tissue sample can be performed, matrix-assisted laser desorption/ionization (MALDI) was introduced. Nevertheless, although offering very good spatial resolution, this method still requires several hours to complete an analysis. ${ }^{6}$ Recently, the concept of an 'intelligent knife' was presented. In this approach, the authors used a mass spectrometer for real-time analysis of volatile compounds present in vapor created by the electric knife used during surgery. ${ }^{7-9}$ Conversely, for non- and semi-volatile analysis of polar compounds, microdialysis (MD) can be used; however, the MD probe needs to be connected with a pump via tubing, requiring additional power supply. This practically restricts the use of MD to single-probe sampling, limiting its exploitability for spatial resolution studies such as drug distribution or simultaneous comparative analysis of different tissue regions, such as tumor vs healthy tissue. On the other hand, MD offers good temporal resolution from constant monitoring of target analytes to collection of dialysate at different time points that ensure detectable amounts of the analytes.

Therefore, there is a gap in the clinical investigation field regarding analysis of semi- and non-volatile broad-range polarity compounds found in tissue with good special and temporal resolution, particularly in real, or close to real time. Such a technique would allow clinicians to make immediate decisions, and take action accordingly. In the current article, we present for the first time the use of in vivo solid-phase microextraction (SPME) for tissue analysis in a clinical environment. The applicability of the method for direct tissue extraction was verified during an experimental animal study using ex vivo lung perfusion (EVLP) and normothermic ex vivo liver perfusion (NEVLP). The details of both approaches to organ preservation before transplantation are described elsewhere. ${ }^{10,11}$

SPME is a technology invented in the early 1990s. It already has an established position as a standard method in environmental and food analysis. The validation of SPME in bioanalysis has been demonstrated for different applications, including determination of drugs, as well as endogenous compounds. ${ }^{12,13}$ Recently, the feasibility of the method was also shown for global screening purposes. ${ }^{14-17}$ The main advantages of in vivo SPME, being a low invasive and nondisturbing method to the system under study, were demonstrated for pharmacokinetics and metabolomics in blood, ${ }^{18-23}$ whereas in vivo tissue analyses were conducted for analysis of pharmaceuticals in living fish. ${ }^{24,25}$ In addition, with the SPME technique, blood sampling can be performed directly from a vein by using a hypodermic needle-assembled SPME probe (Figure 1) or by insertion of the probe to the vein through the i.v. catheter. ${ }^{18}$ This approach can be successfully employed in larger animals (dogs, pigs, and so on) and humans. For small animals such as rodents, because of the small size of their vessels, a number of interfaces facilitating the use of a SPME probe were developed. In addition, to control blood flow through the interface and increase the rate of extraction, a syringe or pump can be connected to the interface. ${ }^{19-23}$ For tissue sampling, the SPME fiber can be placed in the studied tissue either by using the needleassembled device, as it is proposed in this work, or by using specially designed tissue samplers, as reported for fish muscle sampling. ${ }^{25}$ In order to provide fully quantitative results, a

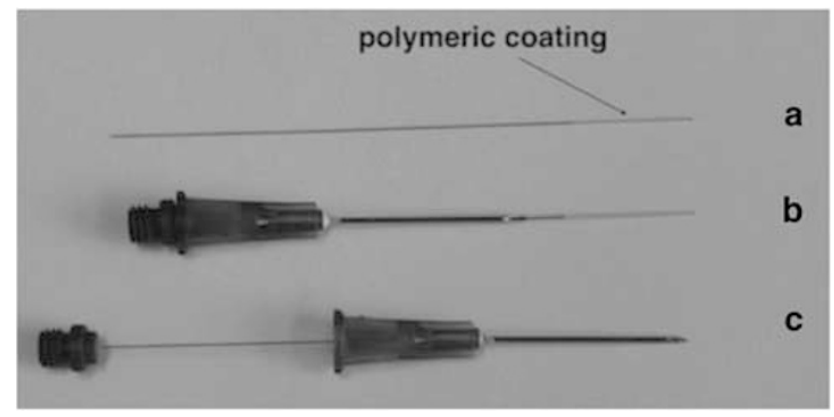

Figure 1 The SPME probe with no assembly (a) and assembled in hypodermic needle $(\mathbf{b}, \mathbf{c})$. The probe shown with exposed polymeric coating used for analyte extraction (b) and with the coating hidden inside the needle for protection during storage and transportation (c). 
number of calibration approaches suitable for various in vivo and on-site applications were developed and validated. ${ }^{26}$ It should also be highlighted that SPME quantitates total as well as free concentrations, thus providing information about the biologically/pharmacologically active fractions of the compound. The experience gained over the past years permits us to propose in vivo SPME as a method for tissue analysis suitable for monitoring of organ assessment, drug concentration and distribution, and biomarker discovery. The results present herein include the proof-of-concept data covering all the aspects mentioned above.

\section{MATERIALS AND METHODS}

Male Yorkshire pigs, $25-35 \mathrm{~kg}$, were utilized for this study. All animals received humane care in compliance with the 'Principles of Laboratory Animal Care' formulated by the National Society for Medical Research and the 'Guide for the Care of Laboratory Animals' published by the National Institutes of Health. All experimental protocols have been approved by the Animal Care Committee at the Toronto General Hospital.

\section{EVLP}

In brief, donor lungs were harvested and preserved at $4{ }^{\circ} \mathrm{C}$, and then transferred to the XVIVO chamber (Vitrolife, Denver, CO, USA). The left atrial (LA) cannula was first connected to the circuit. Flow was initiated slowly in a retrograde manner to de-air the pulmonary artery (PA) cannula. The PA cannula was then connected to the circuit and anterograde flow started at $150 \mathrm{ml} / \mathrm{min}$ with the perfusate (SteenTM Solution, Vitrolife) at room temperature. The temperature of the perfusate was then gradually increased to $37^{\circ} \mathrm{C}$. When $32^{\circ} \mathrm{C}$ was reached (usually over $30 \mathrm{~min}$ ), ventilation was started and the perfusate flow rate was gradually increased. The flow of gas used to deoxygenate and provide carbon dioxide to the inflow perfusate via a gas exchange membrane was then initiated. Mean PA pressures were maintained between 10 and $15 \mathrm{~mm} \mathrm{Hg}$. The LA pressure was maintained between 3 and $5 \mathrm{~mm} \mathrm{Hg}$. Mechanical ventilation setting was: tidal volume of $7 \mathrm{ml} / \mathrm{kg}$, at 7 breaths per min, positive end-expiratory pressure (PEEP) of $5 \mathrm{~cm} \mathrm{H}_{2} \mathrm{O}$ and an inspired oxygen fraction $\left(\mathrm{FiO}_{2}\right)$ of $21 \%$. Detailed information about the technical aspects of the procedure can be found elsewhere. ${ }^{10}$

\section{NEVLP}

A model of donation after cardiac death (DCD) liver retrieval was created by inducing cardiac arrest by potassium chloride at the end of the vascular dissection during the organ retrieval procedure. Briefly, the donor pigs were sedated with ketamine $(0.2 \mathrm{mg} / \mathrm{kg})$, atropine $(0.08 \mathrm{mg} / \mathrm{kg})$, and midazolam $(0.06 \mathrm{mg} / \mathrm{kg})$, and induced with isoflurane $(5 \%)$. Following intubation, the pigs were ventilated with $100 \%$ oxygen and $2 \%$ isoflurane for the duration of the procedure. Laparotomy was then performed, and the infrarenal inferior vena cava
(IVC), aorta, renal arteries, superior mesenteric artery, celiac trunk and hepatic artery (HA), portal vein (PV), suprahepatic IVC, bile duct, and the subdiaphragmatic abdominal aorta were then dissected free and encircled. The gallbladder was removed from the liver. The donor pigs received $30000 \mathrm{IU}$ of heparin in total, $5 \mathrm{~min}$ before cardiac arrest. Following $45 \mathrm{~min}$ of cardiac arrest in all three groups, the organs were flushed with 21 of cold UW solution and stored on ice. The liver was then removed, an arterial back table preparation was performed, 11 of UW solution was infused with pressure into the liver artery, and the liver was stored in cold UW solution. The upper and lower part of the IVC were cannulated with a $1 / 2 \times 3 / 8$ reducer (RAUMEDIC, Helmbrechts, Germany); portal vein and liver artery were cannulated using a $3 / 8 \times 1 / 4$ and $1 / 4 \times 3 / 8$ reducer (RAUMEDIC) in case of subnormothermic ex vivo liver perfusion (SNEVLP). Blood was collected during warm ischemia time (WIT) from the donor. Erythrocytes were isolated by soft spin and stored in CPDA (500 ml, Terumo, Summerset, NJ, USA). After $4 \mathrm{~h}$ of cold ischemic time (CIT), the liver was perfused ex vivo using Steen solution and washed erythrocytes (10\% hematocrit). Three different times of WIT $(0,38$, and $78 \mathrm{~min}$ ) were evaluated. As a control group, $0 \mathrm{~min}$ of WIT was chosen, whereas $38 \mathrm{~min}$ WIT was used to have severe injury in the graft. If the graft remained transplantable and 78 min was used to have an organ suffering from extreme injury, transplantation of these organs would fail in $100 \%$ of the cases. Detailed information about the technical aspects of the procedure can be found in Boehnert et al. ${ }^{11}$

\section{SPME}

\section{Materials and methods}

Acetonitrile, methanol, and water (all LC/MS Optima grade) were purchased from Fisher Scientific (Ottawa, Canada). Prototypes of biocompatible SPME mix-mode probes (C18 with benzenesulfonic acid, $45 \mu \mathrm{m}$ thickness, 4,7 , and $15 \mathrm{~mm}$ length of coating) were provided by Supelco (Bellefonte, PA, USA). The standards used for preparation of instrumental QC samples were purchased from Sigma-Aldrich (D-phenylalanine, L-tryptophan, cholic acid, deoxycholic acid) and $\mathrm{CDN}$ isotopes (L-phenyl-d5-alanine).

\section{In vivo extraction from liver and lung}

Before use, all fibers underwent preconditioning for a minimum of $60 \mathrm{~min}$ of exposure to methanol/water $(1: 1 \mathrm{v} / \mathrm{v})$. In addition, for the lung sampling, sterilization of the fibers was performed by steam autoclaving at $121{ }^{\circ} \mathrm{C}$ for $30 \mathrm{~min}$. The preconcentration mixture (methanol/water, $1: 1 \mathrm{v} / \mathrm{v}$ ) was also sterilized by filtering using $0.45 \mu \mathrm{m}$ pore size sterile syringe filters. The extraction time of lung was $20 \mathrm{~min}$ and liver $30 \mathrm{~min}$. Immediately after sampling, fibers were rinsed with deionized water, dried with Kimwipe and placed on dry ice in the empty vials. After being transported to the laboratory, all fibers were placed at $-80^{\circ} \mathrm{C}$ until analysis. 
Liquid chromatography/mass spectrometry analysis (LC/MS) The samples were analyzed by reverse-phase liquid chromatography (Accela, Thermo Scientific) coupled to bench top orbitrap mass spectrometer (Exactive, Thermo Scientific). The details and conditions of the LC/MS method can be found elsewhere. ${ }^{14,16,17}$

\section{Data processing and statistical analysis}

The raw data files obtained from Thermo Xcalibur were converted to .mzXML using MSConvert software. Subsequently, MZmine software version 2.10 was used for data processing. For filtering, Savitzky-Golay method was used; mass detection was done with exact mass method, using $5 \mathrm{e}^{3}$ noise level. For chromatogram builder, $\mathrm{m} / \mathrm{z}$ tolerance of 0.001 or 5 p.p.m. was used. The chromatogram deconvolution was performed using the Savitzky-Golay method, and for the alignment, the 'join aligner' option was selected. After data processing, the first $\min (0-1 \mathrm{~min})$ corresponding to column void volume and the last $5 \mathrm{~min}$ of column reequilibration (35-40 $\mathrm{min})$ were excluded from the analysis. Given chromatograms were subjected to manual peak picking, and all signals with unacceptable shapes, as well as those present in blank, were removed from the analysis. The final data were exported to a .csv file and used for statistical analysis using SIMCA-P + software version 12.0.1. Pareto scaling was chosen for performing principal component analysis. The statistical confidence level represented as Hotelling $\mathrm{T}^{2}$ ellipse on PCA graphs was $95 \%$. Discriminate metabolites were selected using score contribution plots. The first 10 compounds with the highest absolute contribution score were chosen for further analysis. Putative identification of the compounds selected was performed by comparison of the exact masses obtained from the experimental data with those in the Human Metabolome Database (http://www.hmdb.ca) ${ }^{27}$ with the mass tolerance of 5 p.p.m.

\section{RESULTS \\ Tissue Sampling with SPME Fiber During Surgical Operation}

The approach used for sampling of lung and liver is shown in Figure 2. Sampling of the liver during perfusion using nonassembled and needle-assembled probes is presented in

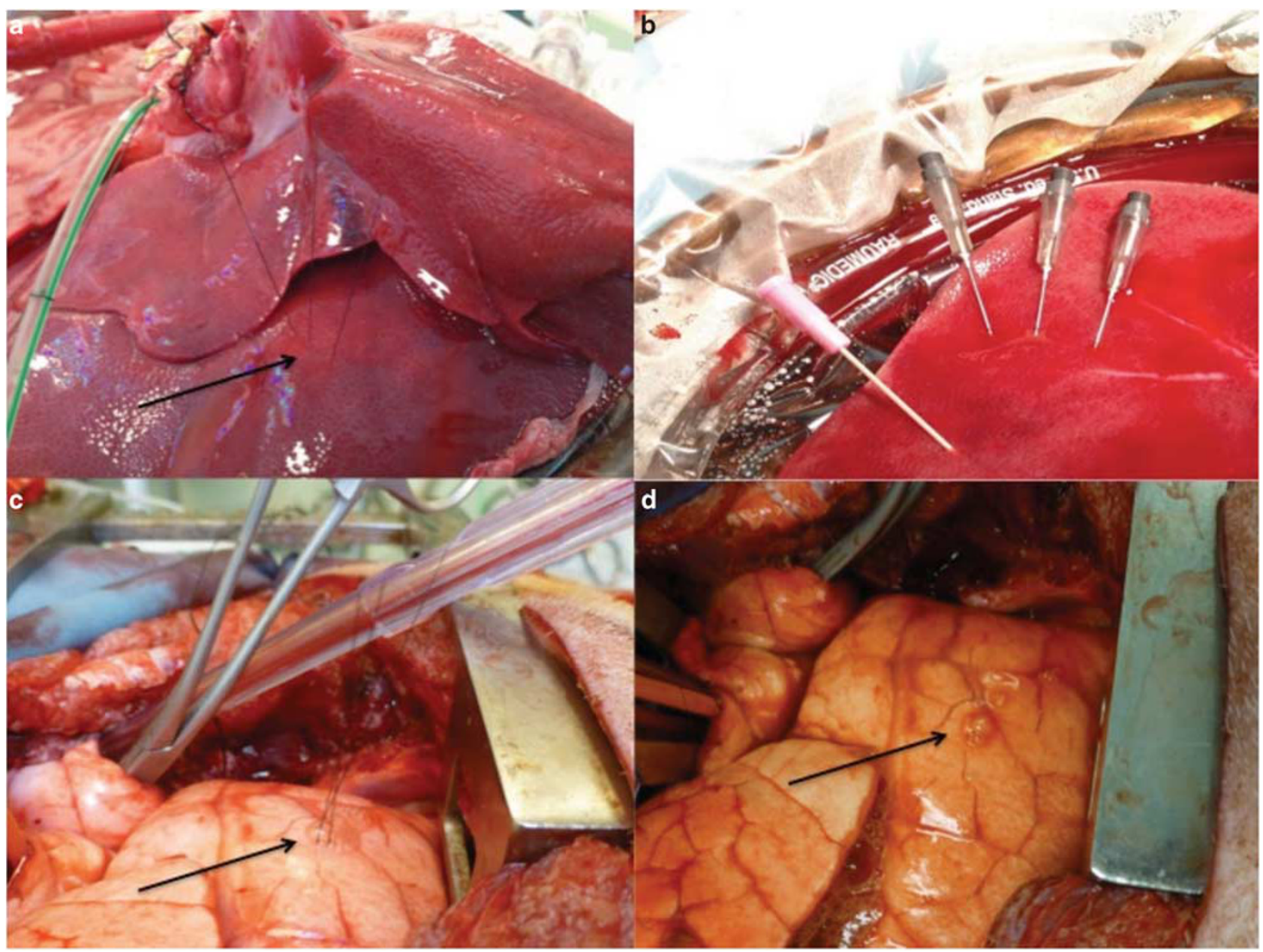

Figure 2 Sampling of organs by SPME with no tissue collection. The in situ extraction of liver with nonassembled (a) and needle-assembled SPME fibers (b), in vivo extraction of lungs with nonassembled SPME fibers (c), and the view of the sampled area after extraction (d). 
Figures $2 \mathrm{a}$ and $\mathrm{b}$, respectively. As a proof of concept, it was also demonstrated that i.v. catheter can be used as a guide cannula for the nonassembled fibers (left part of Figure $2 b$ ). Photos shown in the Figure $2 \mathrm{c}$ and $\mathrm{d}$ were taken during lung transplantation experiments. Figure $2 \mathrm{c}$ shows in vivo extraction with nonassembled fibers that were placed in the tissue with no guide cannula. Figure $2 \mathrm{~d}$ presents lung tissue after SPME probe withdrawal.

\section{To Determine the Drug Level and Metabolism in EVLP System}

To demonstrate the possibility of simultaneous monitoring of drug and products of its metabolism, we have selected methylprednisolone (MP) as a standard medication used during NEVLP and EVLP procedures. The drug was administered as solumedrol, $500 \mathrm{mg}$, in the perfusion solution at the beginning of NEVLP and then $500 \mathrm{mg}$ hourly. To find metabolites of MP, a comprehensive list of potential metabolites of the drug was taken from a recently published article where the authors used a mass spectrometer for identification of products originating from all potential metabolic pathways of MP. ${ }^{28}$ In the current study, the data obtained from liver sampling were analyzed in negative ionization mode and subjected to a target search of MP metabolites. MP was detected in all liver samples that underwent the used preservation protocol, but it was not detected in the sham (Figure 3). The screening of MP metabolites revealed formation of the 17 $\alpha 21$-dihydroxy- $6 \alpha$-methylpregna-1,4-diene-3,11,20-trione, which is the product of 11-oxidation of the parent drug. The metabolite, however, was detected only in the extract from

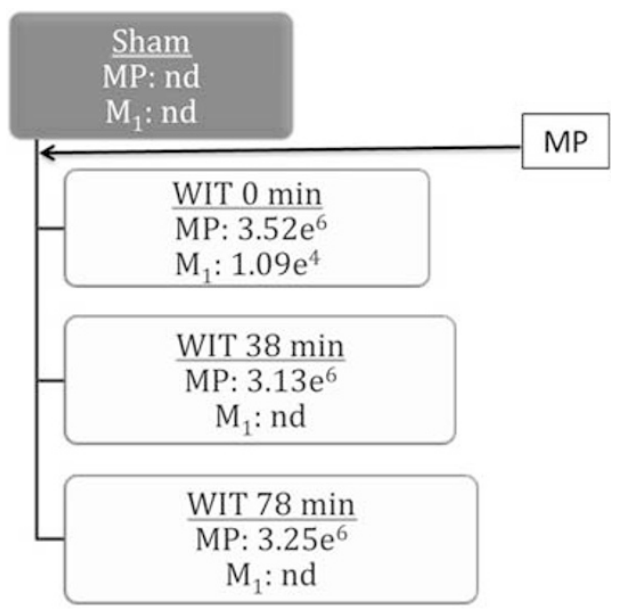

Figure 3 Detection of methylprednisolone (MP) and its metabolite in liver after drug administration during NEVLP. $M_{1}$ indicates 17 $\alpha 21$ dihydroxy-6 $\alpha$-methylpregna-1,4-diene-3,11,20-trione, a metabolite of MP. The scheme presents detection of MP and $M_{1}$ in the extracts obtained from sham liver, and three grafts subjected to different times of warm ischemia time (WIT). The numbers represent average signal intensities of mass spectrometer calculated from three fiber replicates. Relative standard deviation (r.s.d.\%) varied between 3 and $11 \%$. healthy liver (WIT $0 \mathrm{~min}$ ), but not in the injured organs (WIT of 38 and $78 \mathrm{~min}$ ).

\section{Monitoring Changes of Endogenous Metabolites in EVLP System}

Taking into consideration the fact that study of each animal case includes three basic stages, CIT, ex vivo organ perfusion (EVLP/NEVLP), and reperfusion, the data obtained from one entire lung transplantation experiment were subjected to principal component analysis (PCA). The PCA (Figure 4) presents clustering of the lung samples obtained during CIT, EVLP, and reperfusion. As can be noticed, the most distinct differences were observed for the EVLP cluster.

It was not the intention of the authors to draw conclusions of clinical matter, but rather demonstrate the possibilities that the new technology offers in terms of biochemical profiling of living organs. Therefore, selection of the metabolites contributing to differentiation between CIT and EVLP samples was performed and presented as an example. The compounds were tentatively recognized as eicosanoids: prostaglandin F1a; 13,14-Dihydro PGE1; 13,14-Dihydro PGF2a; leukotriene A4; 14,15-EpETE; 5-KETE; 12-HEPE; 14,15-EpETE; 18R-HEPE; 15-HEPE; 17,18-EpETE; 5-HEPE; one of the compounds was identified as main primarily metabolite of melatonin: 6-hydroxymelatonin.

\section{Noninvasive Monitoring Metabolic Changes through Perfusate Sampling}

In addition to tissue sampling, SPME was also used for extraction of analytes from perfusion fluids used in EVLP and NEVLP. To access the fluid standard, a three-way stopcock with luer-lock was connected to the perfusion system (Figure 5). As for a tissue sample, in this case, no sample was collected. As for other matrices, monitoring the changes over time was performed. The evidence of changes occurring with the progression of the lung perfusion as well as the map of molecular features changed over time of EVLP are shown in Figures $6 a$ and $b$, respectively. The labeling of the samples on the PCA plot indicates different sampling time points starting from $0 \mathrm{~h}$ of EVLP through $3 \mathrm{~h}, 6 \mathrm{~h}, 9 \mathrm{~h}$, and to $12 \mathrm{~h}$.

\section{DISCUSSION \\ Tissue Sampling with SPME Fiber During Surgical Operation}

The in vivo sampling of lung and liver using SPME was performed during EVLP and NEVLP. As it can be seen, there are many options of introduction of the SPME probes to the tissue and the format of the device allows for low invasive sampling. The picture presents sampling of liver with the SPME probe nonassembled and assembled in a hypodermic needle (Figures $2 \mathrm{a}$ and $\mathrm{b}$ ) and the sampling of a lung using an unassembled fiber (Figure 2c). The size of the fiber is $<200 \mu \mathrm{m}$, and does not damage the sampled tissue, as it can 


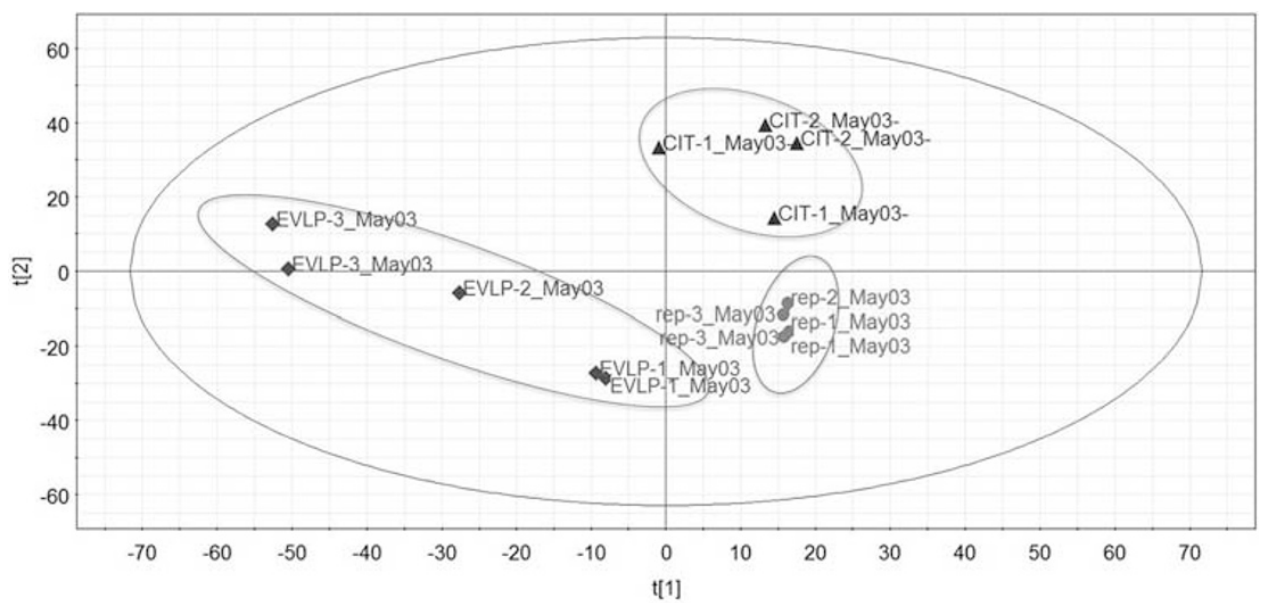

Figure 4 Principal component analysis of samples collected during EVLP. Triangles indicate cold ischemic time, diamonds indicate EVLP, and dots indicate reperfusion.

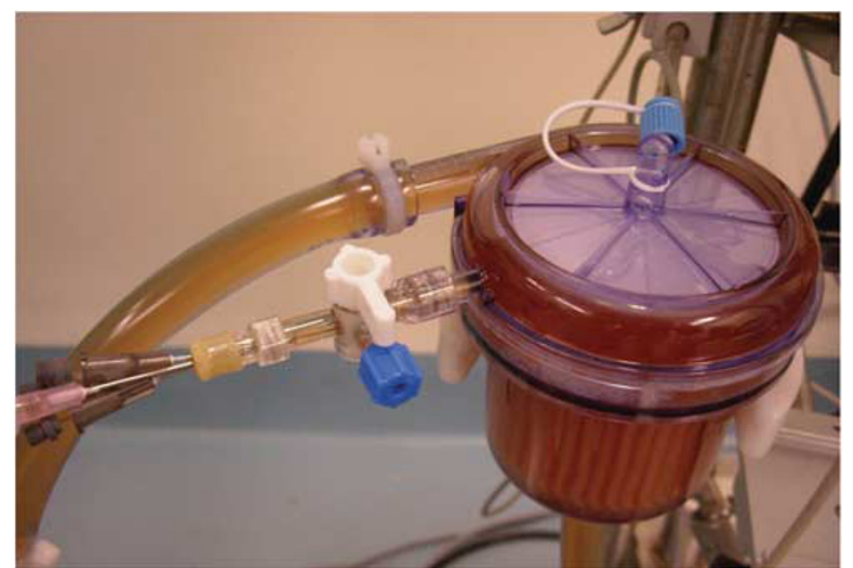

Figure 5 Sampling of perfusate during EVLP using mix-mode SPME probes.

be seen on the picture taken after removing the probe (Figure $2 \mathrm{~d}$ ). The hypodermic needle allows for easier handling of the fiber. Currently, a new, more user-friendly design that permits one-hand operation of the device is under development.

The core of the probe is built from titanium-nickel alloy that makes it very flexible and prevents breaking. The type of SPME probe used for the experiments was a biocompatible co-polymer that permits a wide range of extraction of small metabolites and drugs (molecular weight $<1000 \mathrm{Da}$ ) varying in physiochemical properties. These features of the probe were described previously for direct extraction from human plasma. ${ }^{29}$ The time used for the current sampling approach was 20 and $30 \mathrm{~min}$ for lung and liver, respectively. The fundamentals of SPME as an equilibrium method show that the longer the extraction time, the higher sensitivity of the assay, until the maximum value for a given analyte is reached. Although time of extraction may not be a major concern for in vitrolex vivo applications, with the highest possible sensitivity being achieved by optimum experimental conditions, in real-world application, the sensitivity needs to be compromised, and time of sampling must be adjusted within the medical protocol time. As the overall procedure of organ preservation using ex vivo perfusion lasts several hours, ${ }^{10,11} \mathrm{a}$ sampling time of 20 and $30 \mathrm{~min}$ was considered as a good compromise between sensitivity and temporal resolution.

The SPME probe could also be used for postsurgery monitoring when the fiber is placed inside the examined organ through the guide cannula or catheter, similar to a biopsy needle. The testing of the concept of placing the SPME fiber through the 20 gauge i.v. catheter in the liver was done intrasurgically (Figure 2b), as the insertion of a fiber through the abdominal wall would require a longer catheter and a fiber with a longer wire. This approach will be a subject of future experiments. As expected, the results obtained using the probe inserted via the i.v. catheter were similar to the one obtained with the hypodermic needle assembly.

Despite the small volume of coated polymer, the extraction efficiency of the SPME is comparable with standard methods in terms of analyte coverage and sensitivity. ${ }^{14}$ A metabolomics study of the lung and liver demonstrated the SPME ability of extraction for a wide range of small molecular weight analytes varying in physicochemical properties, including endogenous compounds as well as drugs.

\section{To Determine the Drug Level and Metabolism in EVLP System}

MP was chosen for this proof-of-concept analysis of drug metabolism as it undergoes several biochemical pathways and, as a result, a number of its metabolism products can be expected. Among them, there are metabolites that can be analyzed either in positive or in negative ionization mode, and this was important from the analytical point of view in this study. As mentioned before, solumedrol was adminis- 


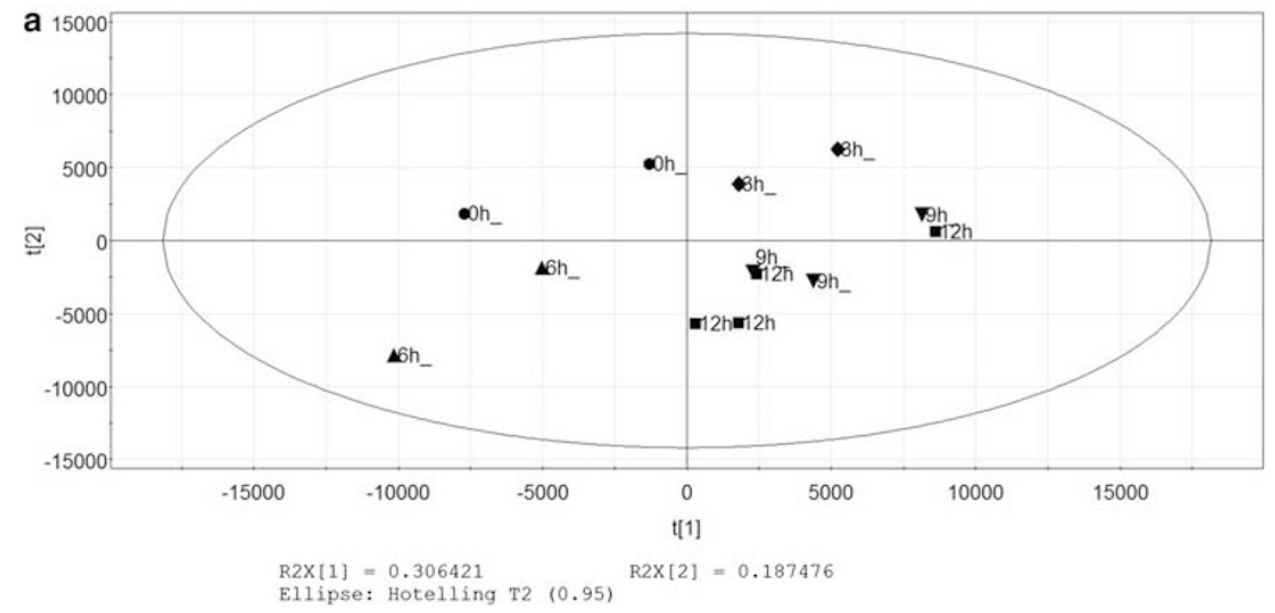

b

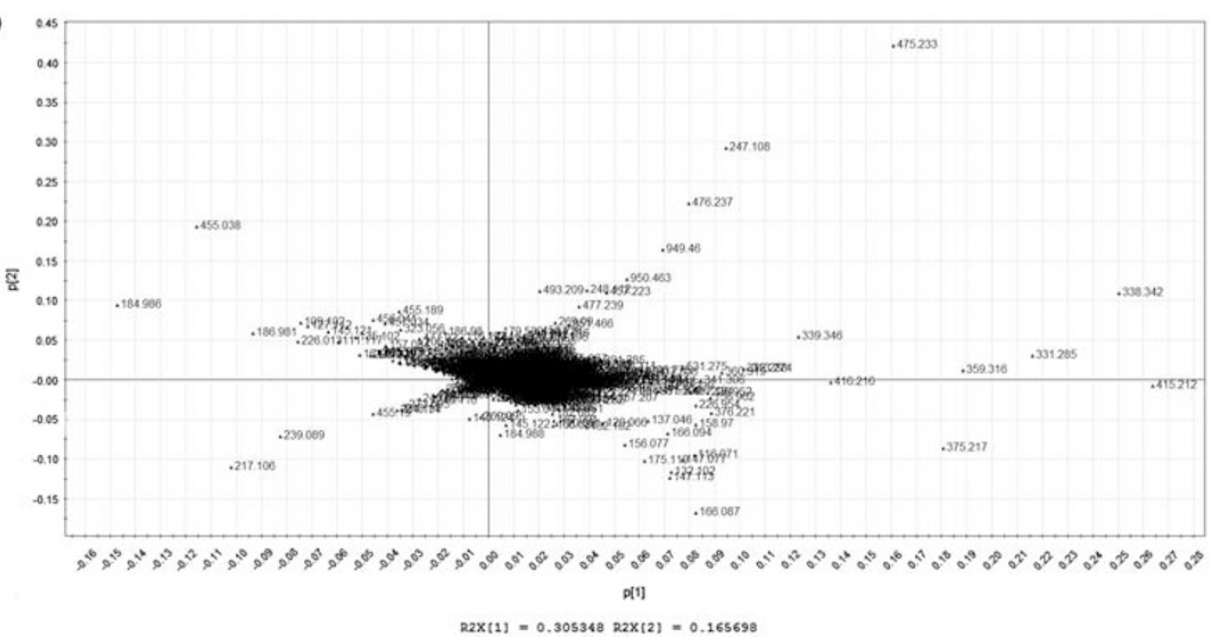

Figure 6 Principal component analysis (a) and loading score plot (b) representing changes occurring during EVLP experiment. Different symbols on PCA plot correspond to different sampling time points: $0 \mathrm{~h}$-dots, $3 \mathrm{~h}$-diamonds, $6 \mathrm{~h}$-triangles, $9 \mathrm{~h}$-inverted triangles, $12 \mathrm{~h}$-boxes.

tered in perfusate, and this explains the absence of the drug in the sham extract and its presence in the liver sampled during NEVLP. The presence of 17 $\alpha 21$-dihydroxy$6 \alpha$-methylpregna-1,4-diene-3,11,20-trione only in the extracts obtained from control (WIT 0 min) may suggest failure of enzymatic function in the cases where liver was subjected to 38 and $78 \mathrm{~min}$ of WIT (Figure 3). This finding was also supported by the alteration of the Krebs and pentose cycles in injured livers; different levels of compounds related to TCA pathways such as oxoglutaric acid, oxalacetic acid, glucaric acid, or galactaric acid, or compounds related to the pentose cycle (D-glucaro-1,4-lactone) were found in healthy and injured livers. The lack of organ recovery in both WIT 38 and WIT 78 min livers can be explained by the fact that SPME sampling was performed at the beginning of the perfusion, and the effect of the procedure on organ regeneration requires a few hours to take effect. Further experiments have been carried out to monitor the progress of the regeneration of enzymatic properties of liver during NEVLP.

\section{Monitoring Changes of Endogenous Metabolites in EVLP System}

As mentioned previously, in vivo sampling with an SPME probe permits metabolic profiling of the organs over time. This feature is the main limitation of the standard techniques, because collecting tissue sample is highly invasive and therefore impossible to apply a number of times. The PCA (Figure 4) obtained on the basis of repetitive sampling of lung during CIT, EVLP, and reperfusion showed clustering of the samples belonging to certain group. The identification of the distinguishing compounds was beyond the scope of this article, but studies using the presented approach are in progress, with a greater number of cases to subsequently demonstrate clinically relevant observations as well as discover biomarkers that could be used in these applications. However, by analysis of the found discriminant metabolites for CIT and EVLP samples, it can be seen that the range of extracted analytes allows to monitor different classes of compounds starting from those with low hydrophobicity such as indoles (6-hydroxymelatonin) to 
lipids (eicosanoids) characterized as strongly hydrophobic species.

\section{Noninvasive Monitoring Metabolic Changes through Perfusate Sampling}

The demonstration of the applicability of SPME to low invasive analysis of tissue was the superior aim of the presented work, as there is currently no alternative method permitting repetitive sample collection-free analysis. However, the technology can be easily extended to different matrices; in the current experimental design, SPME was also used for extraction of perfusates. The extraction time of the perfusate was significantly shorter $(2 \mathrm{~min})$ because the diffusion of compounds in liquids is faster in comparison with solid tissue.

The use of PRN adapter allows for simultaneous sampling of up to three replicates, and this is particularly important in case of short time intervals between the subsequent data points. A similar set up can be used for blood sampling in addition to direct sampling from veins, where the fiber can be placed into the vein through the hypodermic needle. As for the direct organ, microsampling from biofluid permits nondestructive extraction of analytes without interfering with the system equilibrium.

It can be seen on PCA plot obtained for the EVLP experiment (Figure 6a) that during the first $9 \mathrm{~h}$ of the perfusion, position of the samples collected at subsequent sampling points changed, indicating constant variations in the studied matrix, whereas samples representing 9 and $12 \mathrm{~h}$ perfusion time formed one cluster, suggesting that no further changes occurred in the system after $9 \mathrm{~h}$. Figure $6 \mathrm{~b}$ presents the map of compounds characterized by their molecular masses and detected during the described experiments. The metabolites located away from the plot center are the most discriminant features involved in differentiation of the analyzed samples. Among the identified compounds, which changed between the beginning and the end of the EVLP $(0 \mathrm{~h}$ vs $12 \mathrm{~h}$ ), were metabolites involved in biochemical pathways of amino acids, mainly phenylalanine and tyrosine, fatty acids, and their derivatives. More extensive work involving larger cohorts is undergoing.

One of the major requirements for diagnostic instrumentation used for intrasurgical assessment is time of the analysis. As such, the next step of the investigation is the development of a rapid tool for in vivo SPME extraction coupled directly to a mass spectrometer. Several methods of such couplings were already proposed. ${ }^{30-36}$ Current attempts are being made to select the platforms and determine the most efficient method for on-site use. As well, investigations are being conducted to determine the best solution for different groups of compounds and specific biomarkers characterized by different properties. Such approaches would allow results to be obtained in real or close to real time (few minutes), which is the time frame needed by the surgeon to take an immediate action on the course of the operation, or for the anesthesiologist to make changes to the dosage of a drug. The use of this method for preintervention analysis of metabolic profiles could enhance the decision-making process for the best possible personalized approach, whereas posttransplantation monitoring would be used for graft assessments and fast response in case of organ failure.

\section{ACKNOWLEDGMENTS}

We express our sincere gratitude to Dr Marcin Wasowicz for his help in establishing the collaboration between Toronto General Hospital and University of Waterloo. We also thank Ms Nathaly Reyes-Garcés for her help in SPME sampling. The SPME study was supported by Natural Sciences and Engineering Research Council (NSERC) of Canada and Supelco (SigmaAldrich). Supelco (Sigma-Aldrich) provided SPME probes as in kind support. EVLP experiments were supported from SK CIHR (grant 190953 'Molecular Therapeutic Strategies for Ex-vivo Repair of Lungs for Transplantation'), Canadian Cystic Fibrosis Foundation (grant 2387), and SK/ML GL2 (grant GL2-01-019 'Molecular and Genomic Diagnostics to Improve Outcomes in Lung Transplantation'). The funders had no role in study design, data collection and analysis, decision to publish, or preparation of the manuscript.

\section{DISCLOSURE/CONFLICT OF INTEREST}

Janusz Pawliszyn holds the SPME patent, and University of Waterloo receives royalties for sale of SPME device.

1. Nicholson JK, Holmes E, Kinross JM, et al. Metabolic phenotyping in clinical and surgical environments. Nature 2012;491:384-392.

2. Wishart DS. Metabolomics: the principles and potential applications to transplantation. Am J Transplant 2005;5:2814-2820.

3. Masson $P$, Alves AC, Ebbels TM, et al. Optimization and evaluation of metabolite extraction protocols for untargeted metabolic profiling of liver samples by UPLC-MS. Anal Chem 2010;82:7779-7786.

4. Geier FM, Want EJ, Leroi AM, et al. Cross-platform comparison of Caenorhabditis elegans tissue extraction strategies for comprehensive metabolome coverage. Anal Chem 2011;83:3730-3736.

5. Want EJ, Masson P, Michopoulos F, et al. Global metabolic profiling of animal and human tissues via UPLC-MS. Nat Protoc 2013;8:17-32.

6. McDonnell LA, Heeren RM. Imaging mass spectrometry. Mass Spectrom Rev 2007;26:606-643.

7. Schäfer KC, Dénes J, Albrecht K, et al. In vivo, in situ tissue analysis using rapid evaporative ionization mass spectrometry. Angew Chem Int Ed Engl 2009;48:8240-8242.

8. Balog J, Szaniszlo T, Schaefer KC, et al. Identification of biological tissues by rapid evaporative ionization mass spectrometry. Anal Chem 2010;82:7343-7350.

9. Sächfer KC, Szaniszló T, Günther S, et al. In situ, real-time identification of biological tissues by ultraviolet and infrared laser desorption ionization mass spectrometry. Anal Chem 2011;83:1632-1640.

10. Cypel M, Yeung JC, Hirayama S, et al. Technique for prolonged normothermic ex vivo lung perfusion. J Heart Lung Transplant 2008;27: 1319-1325.

11. Boehnert MU, Yeung JC, Bazerbachi F, et al. Normothermic acellular ex vivo liver perfusion reduces liver and bile duct injury of pig livers retrieved after cardiac death. Am J Transplant 2013;13:1441-1449.

12. Bojko B, Cudjoe E, Gómez-Ríos GA, et al. SPME-quo vadis? Anal Chim Acta 2012;750:132-151.

13. Bojko B, Cudjoe E, Wasowicz $M$, et al. Solid-phase microextraction How far are we from clinical practice? TrAC Trends Anal Chem 2011;30:1505-1512.

14. Vuckovic D, de Lannoy I, Gien B, et al. In vivo solid-phase microextraction: capturing the elusive portion of metabolome. Angew Chem Int Ed Engl 2011;50:5344-5348.

15. Vuckovic D, Risticevic S, Pawliszyn J. In vivo solid-phase microextraction in metabolomics: opportunities for the direct investigation of biological systems. Angew Chem Int Ed Engl 2011;50: 5618-5628.

16. Bessonneau V, Bojko B, Pawliszyn J. Analysis of human saliva metabolome by direct immersion solid-phase microextraction LC and benchtop orbitrap MS. Bioanalysis 2013;5:783-792. 
17. Bojko B, Wasowicz M, Pawliszyn J. Metabolic profiling of plasma from cardiac surgical patients concurrently administered with tranexamic acid. DI-SPME-LC-MS analysis. J Pharm Anal 2014;4:6-13.

18. Lord HL, Grant RP, Walles M, et al. Development and evaluation of a solid-phase microextraction probe for in vivo pharmacokinetic studies. Anal Chem 2003;75:5103-5115.

19. Musteata FM, Musteata ML, Pawliszyn J. Fast in vivo microextraction: a new tool for clinical analysis. Clin Chem 2006;52:708-715.

20. Musteata FM, De Lannoy I, Gien B, et al. Blood sampling without blood draws for in vivo pharmacokinetic studies in rats. J Pharm Biomed Anal 2008;47:907-912.

21. Musteata FM, Pawliszyn J. In vivo sampling with solid phase microextraction. J Biochem Biophys Methods 2007;70:181-193.

22. Lord $\mathrm{HL}$, Zhang $\mathrm{X}$, Musteata FM, et al. In vivo solid-phase microextrac tion for monitoring intravenous concentrations of drugs and metabolites. Nat Protoc 2011;6:896-924.

23. Vuckovic D, de Lannoy I, Gien B, et al. In vivo solid-phase microextraction for single rodent pharmacokinetics studies of carbamazepine and carbamazepine-10, 11-epoxide in mice. J Chromatogr A 2011;1218:3367-3375.

24. Cudjoe $E$, Bojko B, Togunde $P$, et al. In vivo solid-phase microextraction for tissue bioanalysis. Bioanalysis 2012;4:2605-2619.

25. Togunde OP, Lord H, Oakes KD, et al. Development and evaluation of a new in vivo solid-phase microextraction sampler. J Sep Sci 2013:36:219-223.

26. Ouyang G, Pawliszyn J. A critical review in calibration methods for solid-phase microextraction. Anal Chim Acta 2008;627:184-197.

27. Human Metabolome Database. http://www.hmdb.ca. accessed April 2013.

28. Pozo OJ, Marcos J, Matabosch X, et al. Using complementary mass spectrometric approaches for the determination of methylprednisolone metabolites in human urine. Rapid Comm Mass Spectrom 2012:26:541-553.
29. Vuckovic D, Pawliszyn J. Systematic evaluation of solid-phase microextraction coatings for untargeted metabolomic profiling of biological fluids by liquid chromatography-mass spectrometry. Anal Chem 2011;83:1944-1954.

30. Mirnaghi FS, Pawliszyn J. Reusable solid-phase microextraction coating for direct immersion whole-blood analysis and extracted blood spot sampling coupled with liquid chromatography-tandem mass spectrometry and direct analysis in real time-tandem mass spectrometry. Anal Chem 2012;84:8301-8309.

31. Wang $Y$, Walles $M$, Thomson $B$, et al. Solid-phase microextraction combined with surface-enhanced laser desorption/ionization introduction for ion mobility spectrometry and mass spectrometry using polypyrrole coatings. Rapid Comm Mass Spectrom 2004;18: 157-162.

32. Perera S, Berthod A, Dodbiba E, et al. Coupling solid-phase microextraction and laser desorption ionization for rapid identification of biological material. Rapid Comm Mass Spectrom 2012;26: 853-862.

33. Lokhnauth JK, Snow NH. Solid phase micro-extraction coupled with ion mobility spectrometry for the analysis of ephedrine in urine. J Sep Sci 2005;28:612-618.

34. Tong $\mathrm{H}$, Sze N, Thomson B, et al. Solid phase microextraction with matrix assisted laser desorption/ionization introduction to mass spectrometry and ion mobility spectrometry. Analyst 2002;127: 1207-1210.

35. Walles M, Gu Y, Dartiguenave C, et al. Approaches for coupling solidphase microextraction to nanospray. J Chromatogr A 2005;1067: 197-205.

36. Rodriguez-Lafuente A, Mirnaghi FS, Pawliszyn J. Determination of cocaine and methadone in urine samples by thin-film solid-phase microextraction and direct analysis in real time (DART) coupled with tandem mass spectrometry. Anal Bioanal Chem 2013;405: 9723-9727. 